\title{
An Attentional Recurrent Neural Network for Personalized Next Location Recommendation
}

\author{
Qing Guo, ${ }^{1}$ Zhu Sun, ${ }^{2}$ Jie Zhang, ${ }^{3}$ Yin-Leng Theng ${ }^{4}$ \\ 1,2,3,4 Nanyang Technological University, Singapore \\ 1,2,3,4 \{qguo006, zhu.sun, zhangj, tyltheng\}@ntu.edu.sg
}

\begin{abstract}
Most existing studies on next location recommendation propose to model the sequential regularity of check-in sequences, but suffer from the severe data sparsity issue where most locations have fewer than five following locations. To this end, we propose an Attentional Recurrent Neural Network (ARNN) to jointly model both the sequential regularity and transition regularities of similar locations (neighbors). In particular, we first design a meta-path based random walk over a novel knowledge graph to discover location neighbors based on heterogeneous factors. A recurrent neural network is then adopted to model the sequential regularity by capturing various contexts that govern user mobility. Meanwhile, the transition regularities of the discovered neighbors are integrated via the attention mechanism, which seamlessly cooperates with the sequential regularity as a unified recurrent framework. Experimental results on multiple real-world datasets demonstrate that ARNN outperforms state-of-the-art methods.
\end{abstract}

\section{Introduction}

With the advancement of mobile technologies, locationbased social network (LBSN) services (e.g., Foursquare, Facebook place and Yelp) have become increasingly more popular in recent years. Next location recommendation in LBSNs is an important function as it enables users to explore more interesting locations and better plan their trips (Cheng et al. 2013; Liu et al. 2016; Feng et al. 2018). Existing approaches mostly focus on capturing the sequential regularity for modeling human mobility (Liu et al. 2016; Feng et al. 2018; Tang and Wang 2018), i.e., users' next movement highly relates to previously visited locations. Yet next location recommendation in LBSNs is still very difficult due to the extreme sparsity issue (Yao et al. 2017; Feng et al. 2018; Sun et al. 2019) - our data analysis shows that most locations are only followed by fewer than 5 locations consecutively. Such data sparsity issue makes it hard to learn effective sequential patterns among locations.

To solve the sparsity problem, we leverage the transition regularities of similar locations (neighbors) by assuming

Copyright (c) 2020, Association for the Advancement of Artificial Intelligence (www.aaai.org). All rights reserved.

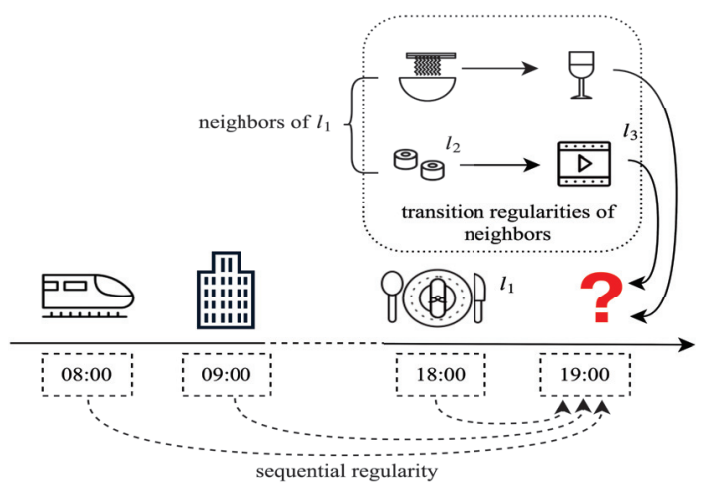

Figure 1: A running example

that they share similar transitional patterns. Fig. 1 presents a running example to illustrate our intuition. $l_{1}$ is an Italian restaurant followed by only a few locations, so it is challenging to predict the next location that a user might visit after $l_{1}$ by only capturing the sequential regularity. In fact, the transitional information of $l_{1}$ 's neighbors can help ease such sparsity issue. For instance, if users often watch a film at a cinema $l_{3}$ after having dinner at $l_{2}\left(l_{1}\right.$ 's neighbor which is a nearby Sushi restaurant), then $l_{3}$ can also be recommended as the next location for $l_{1}$. In other words, the transition regularities of neighbors serve as complementary information for locations with insufficient following check-ins. Finally, the transition regularities of neighbors at each time step can cooperate with the sequential regularity of previous check-in sequence to jointly predict users' next movement.

Due to the heterogeneous nature of LBSNs, multiple factors make connections between two locations including geographical distance, semantics (POI categories and tags) and user preference (Xie et al. 2016; Guo et al. 2017; Yin et al. 2017). We expand similar locations by assuming two locations are similar if they are: 1) geographically close; 2) described by the same semantics; 3 ) preferred by the same user. Our idea can be considered as a neighborhood-based strategy, i.e., aggregating useful features of neighbors for recommendation. To our best knowledge, we are the first to solve such data sparsity issue by coherently modeling the se- 
quential regularity with the transition regularities of neighbors for next location recommendation.

To jointly model both regularities, we propose a novel Attentional Recurrent Neural Network framework (ARNN), which seamlessly integrates RNN (Hochreiter and Schmidhuber 1997) and attention mechanism (Bahdanau, Cho, and Bengio 2014) in a unified framework. A multi-modal embedding layer is first adopted to transform the sparse features of each check-in in the sequence into dense representations that are further fed into the recurrent layer. To extract various types of neighbors, we construct a novel knowledge graph $(\mathrm{KG})$ to accommodate the heterogeneous information including users, locations and semantics (categories and tags of locations) into a unified space. A meta-path based random walk process over $\mathrm{KG}$ is then designed to efficiently discover the neighbors based on geographical, semantic and user preference factors. Next, we propose a novel method of using attention mechanism to capture the transition regularities of neighbors. Specifically, the attention layer is capable of selecting highly salient neighbors that are positively correlated to the current location at each time step. Finally, the attention layer is tailored to effectively cooperate with the recurrent layer in a unified manner. The experimental results on multiple LBSN datasets show that ARNN outperforms state-of-the-art algorithms, with significant improvements of accuracy by $9.21 \%$ on average.

\section{Related Work}

There are two types of methods for user next movement prediction - pattern-based and model-based methods (Yao et al. 2017). Pattern-based methods can only mine explicitly pre-defined patterns on dense trajectories without capturing all mobility regularities about user movement (Monreale et al. 2009; Li et al. 2010). Thus, they are not suitable for LBSN scenario with extremely sparse trajectory data. In contrast, model-based methods are favored in LB$\mathrm{SNs}$, due to their ability to model complex movement regularities and fuse heterogeneous data (He et al. 2016; Tang and Wang 2018). Hence, we mainly discuss state-ofthe-art model-based methods that can be further classified into Markov-based and NN-based methods.

\section{Markov-based Models}

Generally, Markov-based models (MMs) predict the probability of future visit by constructing a location transition matrix. Due to the sparse check-in data, latent factor model (Koren, Bell, and Volinsky 2009) is always adopted to help learn dense representations. By combining MMs with matrix factorization (MF) (Mnih and Salakhutdinov 2008), Rendle et al. (2010) propose FPMC to model user sequential behavior at personalized level. Cheng et al. (2013) extend FPMC to learn transition regularities with localized spatial constraint. He et al. (2016) incorporate temporal and categorical information via a weighting scheme based on firstorder Markov chain property.

However, MMs aim to learn transition probability between successive locations, thus failing to capture highorder sequential regularity. Besides, existing MMs fuse different contexts in a linear fashion such that impacts of those contexts cannot be well captured. Instead, we adopt the recurrent neural network, which can model the long-term sequences and flexibly fuse the diverse contextual information at each time step via the multi-modal embedding layer.

\section{NN-based Models}

Recent studies apply Neural Network (NN) in next location recommendation, thanks to its strong capability of capturing sequential information (Hochreiter and Schmidhuber 1997; Mikolov et al. 2010; Zhang et al. 2014; Huang et al. 2019). By leveraging the similar users, Massimo and Ricci (2018) propose an approach based on Inverse Reinforcement Learning (IRL) that can allow users to maximise their estimated reward (utility) by visiting the suggested POIs. By using the attention mechanism, Huang et al. (2019) propose an attention-based LSTM model (ATST-LSTM) that can focus on the relevant historical check-in records in a checkin sequence by resembling the sequence to sequence model in machine translation (Sutskever, Vinyals, and Le 2014). To better capture the multi-level periodicity, Feng et al. (2018) propose DeepMove by fusing an attention module into Recurrent Neural Network (RNN), to automatically select highly correlated historical records for current status. Caser adopts Convolutional Neural Network (CNN) to capture the joint effects of previous check-ins on the current check-in (Tang and Wang 2018). This enables Caser to advance DeepMove, as DeepMove models the impact of each check-in independently.

Another research line explores new methods of using contextual information. Some studies model human mobility by using temporal and spatial contexts via time and distance transition matrices (Liu et al. 2016; Zhang et al. 2017). However, the model training is complicated by the heavy parameters. SERM jointly learns the embeddings of multiple contexts (e.g., temporal and semantic information) with user preference (Yao et al. 2017). It is more flexible to incorporate various contexts with fewer parameters involved. However, only limited improvements are achieved by modeling both sequential regularity and multiple contexts, as these methods still cannot generate decent recommendations for the locations with only few following records. Therefore, we propose a novel recurrent framework (ARNN) to exploit the transition regularities of neighbors based on the heterogeneous contextual factors to overcome the sparsity challenge.

\section{Problem Analysis and Formulation}

\section{Problem Analysis}

We adopt several real-world datasets from Foursquare (Yang et al. 2015) and Gowalla ${ }^{1}$. As most successive check-ins happen within 10km (Cheng et al. 2013; Feng et al. 2015), we choose three major cities for the data analysis and experiments: New York (NY) and Tokyo (TK) from Foursquare and San Francisco (SF) from Gowalla. Foursquare APIs ${ }^{2}$ are applied to collect categories and tags of locations in SF.

\footnotetext{
${ }^{1}$ https://snap.stanford.edu/data/loc-gowalla.html

${ }^{2} \mathrm{https}$ ///developer.foursquare.com/places-api
} 


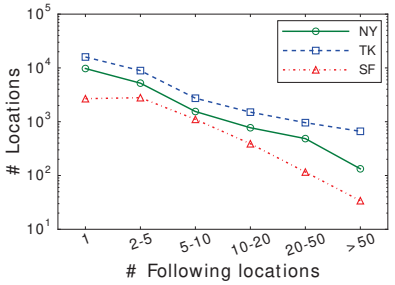

(a) Three cities

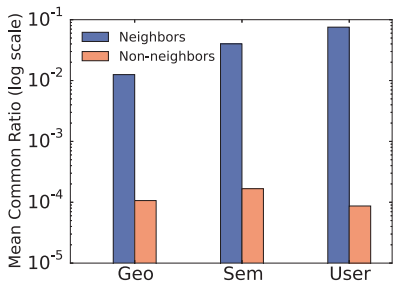

(b) New York

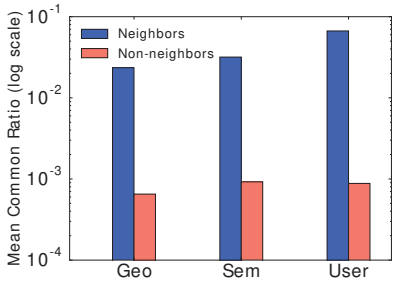

(c) Tokyo

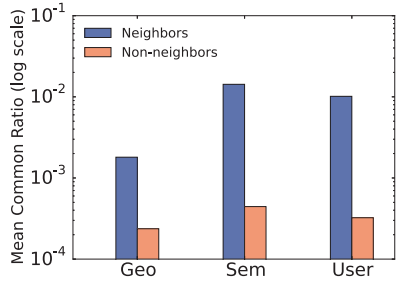

(d) San Fransisco

Figure 2: Fig. 2(a) reports the distribution of locations w.r.t. \# following locations; Fig. 2(b-d) presents the mean common ratio of neighbors vs. non-neighbors based on the three factors, including geographical, semantic and user preference factors.

Sparsity Issue. To assess the sparsity, for each location $l_{k}$, we calculate the number of its following locations. A following location of $l_{k}$ is defined as the location visited after $l_{k}$ successively. Fig. 2(a) shows that most locations have a very limited number of following locations. In particular, over $50 \%$ locations have only one following location in $\mathrm{NY}$ and TK while more than $80 \%$ have fewer than 5 following locations in all three cities. Such insufficiency of following locations create extreme hardness to learn the transitional patterns between locations to predict the next movement.

Transition Regularities of Neighbors. To quantitatively validate our assumption on transition regularities of neighbors, we calculate the common ratio about the following locations for a given location pair $-l_{k}$ and $l_{j}$. Let $L_{f}\left(l_{k}\right)$ and $L_{f}\left(l_{j}\right)$ denote the sets of following locations of $l_{k}$ and $l_{j}$, respectively. The common ratio measures the overlap degree of $L_{f}\left(l_{k}\right)$ and $L_{f}\left(l_{j}\right)$ defined as: $\alpha\left(l_{k}, l_{j}\right)=\frac{\left|L_{f}\left(l_{k}\right) \cap L_{f}\left(l_{j}\right)\right|}{\left|L_{f}\left(l_{k}\right) \cup L_{f}\left(l_{j}\right)\right|}$. Based on our intuition, if $l_{k}$ is similar to $l_{j}, L_{f}\left(l_{k}\right)$ and $L_{f}\left(l_{j}\right)$ should have more overlap. We calculate the mean common ratios of three types of neighbors w.r.t. geographical (Geo), semantic (Sem) and user preference (User) factors compared with non-neighbors. Fig. 2(b-d) show that the mean common ratios of neighbors are consistently much higher than non-neighbors. This verifies that similar locations share more similar transitional patterns. Therefore, aggregating the transition regularities of neighbors can help ease the sparsity issue for high-quality recommendation performance.

\section{Problem Formulation}

To formulate the problem, let $U=\left\{u_{1}, u_{2}, \ldots, u_{|U|}\right\}, L=$ $\left\{l_{1}, l_{2}, \ldots, l_{|L|}\right\}$ and $V=\left\{w_{1}, w_{2}, \ldots, w_{|V|}\right\}$ denote a set of users, locations and words (POI categories and tags), respectively.

Definition 1. Historical Sequence. The historical sequence of user $u_{i}$ is a temporally ordered sequential check-in records, i.e., $\operatorname{His}\left(u_{i}\right)=\left\{r_{1}, r_{2}, \ldots, r_{H}\right\}$. And each check-in $r_{k} \in \operatorname{His}\left(u_{i}\right)$ is a tuple $\left(u_{i}, l_{k}, t_{k}\right)$ where $l_{k}$ is the location and $t_{k}$ is the timestamp. $S\left(l_{k}\right) \subset V$ is the semantics of $l_{k}$ including a bag of words (the categories and tags of $l_{k}$ ).

Definition 2. Trajectory. A trajectory of a user $u_{i}$ is a subsequence of $\operatorname{His}\left(u_{i}\right)$ where the time interval between two suc-

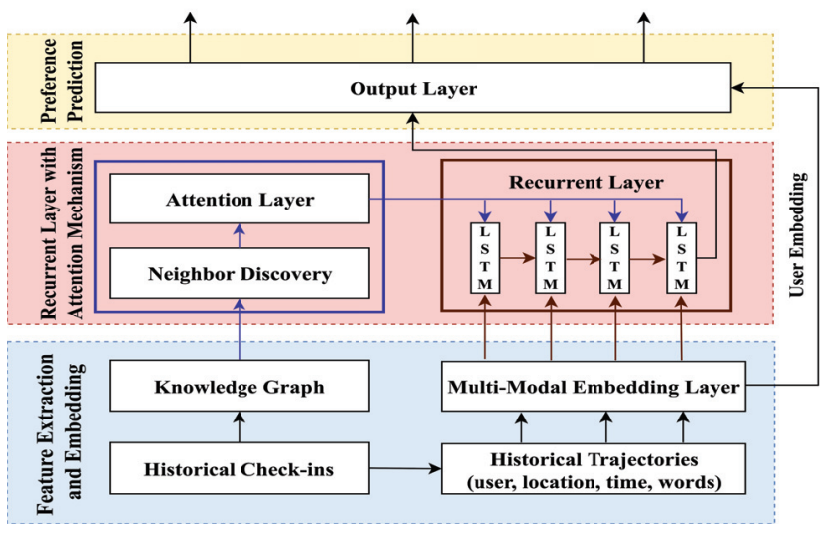

Figure 3: The architecture of ARNN

cessive check-ins is smaller than the pre-defined time threshold, $\Delta t$, i.e., $\operatorname{Tra}\left(u_{i}\right)=\left\{r_{1}, r_{2}, \ldots, r_{M}\right\}$ is a segment of $H i s\left(u_{i}\right)$, if $0<t_{m+1}-t_{m} \leqslant \Delta t, \forall 1 \leqslant m<M$.

The next location recommendation is formulated as: given a trajectory of user $u_{i}$, i.e., $\operatorname{Tr} a\left(u_{i}\right)=\left\{r_{1}, r_{2}, \ldots, r_{K-1}\right\}$, a list of locations that $u_{i}$ would visit at time step $t_{K}$ are generated. As the following location of $l_{k}$ is highly sparse, i.e., $\left|L_{f}\left(l_{k}\right)\right|$ is always fewer than 5, we thus leverage transition regularities of neighbors at each time step to ease such sparsity issue for better recommendation.

\section{The Proposed Framework}

Our proposed Attentional Recurrent Neural Network framework (ARNN) consists of four layers: embedding layer, attention layer, recurrent layer and output layer, depicted in Fig. 3. We first design a multi-modal embedding layer to learn the dense representations of locations and various contexts. To find relevant neighbors, a novel knowledge graph $(\mathrm{KG})$ is built by fusing the heterogeneous data into a unified space. Then, a meta-path based random walk over the KG is designed to efficiently discover the neighbors based on multiple factors. To capture the transition regularities of relevant neighbors, an attention layer is developed to generate a weighted embedding by distinguishing each neighbor of the current location. By integrating the weighted embedding from attention layer and current status, the recurrent layer 


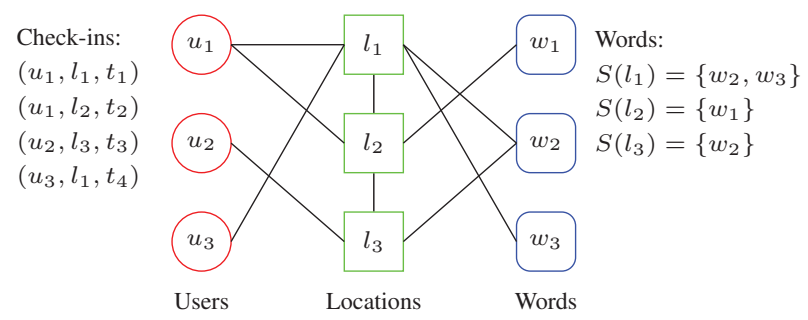

Figure 4: An illustrative example of knowledge graph

can generate the hidden state that encodes the information observed until the current time step. Finally, the output layer jointly considers both the information of the trajectory and user general interest to recommend the next location.

\section{Neighbor Discovery}

To capture the heterogeneous factors that enable the connections among locations, we leverage the knowledge graph and meta-path for neighbor discovery. First, a novel knowledge graph is constructed, i.e., $G=\left\{U \cup L \cup V, E_{U L} \cup\right.$ $\left.E_{L L} \cup E_{L V}\right\}$, where $E_{L L}$ represents location-location relations, that is, two locations are linked if they are geographically close to each other, i.e., $\operatorname{dist}\left(l_{k}, l_{j}\right) \leqslant \Delta d$ where $\Delta d$ is a distance threshold; $E_{L V}$ denotes location-word affiliations; $E_{U L}$ denotes user historical visits. Each input tuple $\left(u_{i}, l_{k}, t_{k}\right)$ denotes a check-in, $r_{k}$, while each location is described by a set of words, $S\left(l_{k}\right)$. By aggregating the historical check-ins, the words of locations, and geographical distance between locations, $G$ is capable of accommodating the heterogeneous data of historical records into a unified representation space as illustrated in Fig. 4.

Next, we utilize meta-path, which is a critical technique to capture diverse semantic relations in a heterogeneous network (Sun et al. 2011), to extract various kinds of neighbors from $G$. Specifically, a meta-path is represented by a sequence of linked entities at the scheme level, e.g., metapath $L U L$ can build bridges for two locations visited by the same users. Based on our analysis in Fig. 2 (b-d), three meta-paths, $L L, L V L$ and $L U L$, are selected, representing geographical, semantic and user preference factors, respectively. For each location, a random walk process is conducted to generate paths based on the above meta-paths. Then, the neighbors are extracted from those paths. The random walk process is illustrated as follows. A meta-path is defined as $p=T_{1} T_{2} \cdots T_{m} \cdots$, where $T_{m}$ is the type of $m$-th entity. The transition probability between two linked entities is determined by the neighborhood size with constraint based by $p$, i.e., $\operatorname{Prob}\left(v_{m} \mid v_{m-1}, p\right)=\frac{1}{\left|N_{T_{m}}\left(v_{m-1}\right)\right|}$, if $T\left(v_{m-1}\right)=T_{m-1}$ and $T\left(v_{m}\right)=T_{m}$, where $T(v)$ is the type of entity $v$ and $N_{T_{m}}(v)$ is the first-order neighbor set of $v$ in type $T_{m}$. By following $p$ with the transition probability, the random walker could generate a path until it reaches the walk length. The process terminates if enough paths are created. Finally, we extract locations from those paths to create the neighbor set of a given location. For example, we have $l_{1} \rightarrow w_{2} \rightarrow l_{3}$ in Fig. 4 , so $l_{3} \in N_{p}\left(l_{1}\right)$ where $p=L V L$ and $N_{p}\left(l_{1}\right)$ is the neighbor set of $l_{1}$ w.r.t. meta-path $p$. Algo-

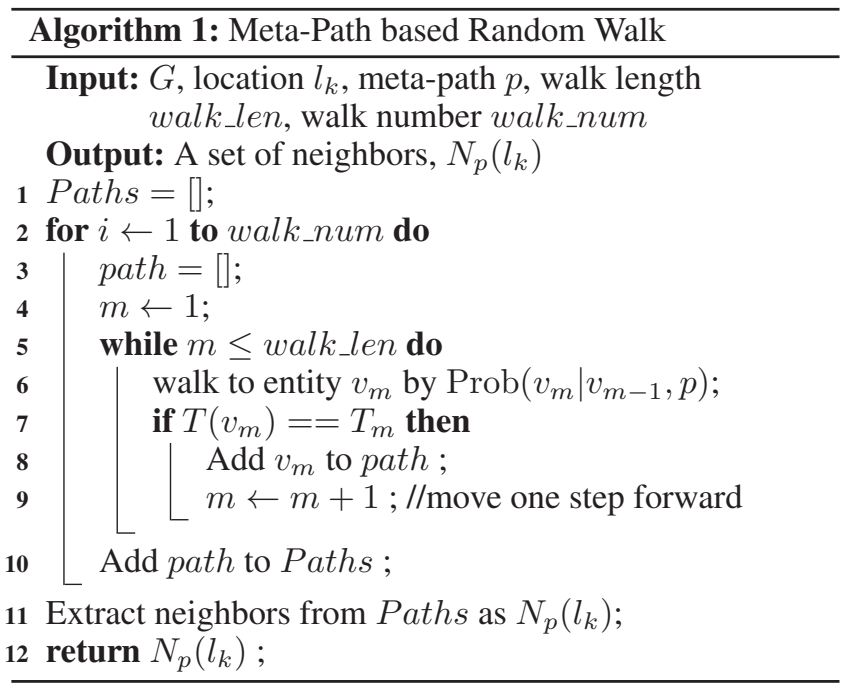

rithm 1 describes the details about the random walk process.

\section{Embedding Layer}

A multi-modal embedding layer is devised to jointly learn the embeddings of the location with its temporal context, semantic context, and user general interest. Specifically, different information of each check-in is initially represented as one-hot vectors. As the timestamp is a continuous value, we then map one day into 24 hours so that $t_{k}$ can be transformed into 24-dimensional one-hot vector. Each location is represented by a $|L|$-dimensional one-hot vector. For the semantics of each location, we first transfer each word into a $|V|$-dimensional one-hot vector, and sum them up as a new vector. Besides, we assume each user has her general preference, represented as a $|U|$-dimensional one-hot vector. These one-hot vectors are fed into the embedding layer to learn the low-dimensional dense representations of the timestamp, location, semantics and user general interest, denoted as $\boldsymbol{e}_{t} \in \mathbb{R}^{D_{t}}, \boldsymbol{e}_{l} \in \mathbb{R}^{D_{l}}, \boldsymbol{e}_{s} \in \mathbb{R}^{D_{s}}$ and $\boldsymbol{e}_{u} \in \mathbb{R}^{D_{u}}$, respectively. Note, unlike other embeddings, user embedding, $\boldsymbol{e}_{u}$, is not taken as an input into the recurrent layer, but only used by the output layer at the last time step of the trajectory, as it is considered to be stable through time (Yao et al. 2017; Feng et al. 2018; Tang and Wang 2018). These dense representations can model the semantic and spatio-temporal features of each check-in more precisely as well as reduce the computation.

\section{Attention Layer}

To model the transition regularities of neighbors, we exploit the attention mechanism (Mnih et al. 2014) to choose salient neighbors based on current location automatically at each time step along the sequence. Specifically, the attention layer is designed to calculate the similarity (i.e., attention weights) between current location and each neighbor. If a neighbor is more similar with current location w.r.t. transition patterns, it would be assigned with a larger attention weight. Besides, the attention layer is parametrized as a feed-forward neural 
network, which is jointly trained with other layers. Fig. 3 also illustrates the structure of the attention layer. The following describes the attention computation at time step $t_{k}$ :

$$
\begin{aligned}
& \boldsymbol{c}_{k}=\sum_{n} \boldsymbol{\alpha}_{k}(n) \boldsymbol{e}_{l_{n}}, \\
& \boldsymbol{\alpha}_{k}(n)=\operatorname{softmax}\left(f_{a}\left(\boldsymbol{e}_{l_{k}}, \boldsymbol{e}_{l_{n}}\right)\right), \\
& f_{a}\left(\boldsymbol{e}_{l_{k}}, \boldsymbol{e}_{l_{n}}\right)=\tanh \left(\boldsymbol{e}_{l_{k}}^{\top} \cdot \boldsymbol{W}_{a} \cdot \boldsymbol{e}_{l_{n}}\right)
\end{aligned}
$$

where $e_{l_{k}}$ is the embedding of current location; $\boldsymbol{e}_{l_{n}}$ is the embedding of location $l_{n}$, a neighbor of $l_{k} ; c_{k} \in \mathbb{R}^{D_{l}}$ is computed as the weighted average embedding over all the neighbors; $\boldsymbol{W}_{a}$ is the weight matrix; $\boldsymbol{\alpha}_{k}(n) \in[0,1]$ is the attention weight of $l_{n}$ and $\sum_{n} \boldsymbol{\alpha}_{k}(n)=1 ; f_{a}$ is the score function, which measures the relevance between the neighbor and current location.

\section{Recurrent Layer}

To capture the high-order sequential regularity, we adopt Long Short-Term Memory (LSTM) as the recurrent unit, due to its strong capability of memorizing long-range sequential information (Hochreiter and Schmidhuber 1997). At time step $t_{k}$, we have the representations generated by different components of ARNN, including: 1) $e_{l_{k}}, e_{t_{k}}, e_{s_{k}}$ from the multi-modal embedding layer, which embed current location, its temporal and semantic contexts; 2) $\boldsymbol{c}_{k}$ from the attention layer, which represents the weighted embedding based on relevant neighbors; 3) $\boldsymbol{h}_{k-1}$ from previous recurrent unit, which encodes the information of the trajectory until $t_{k-1}$. The goal of the recurrent unit is to jointly integrate the three groups of representations to update the hidden state, which preserves the information observed until the current time step $t_{k}$. First, they are concatenated to generate a new representation, i.e., $\boldsymbol{e}_{k}=\left[\boldsymbol{e}_{l_{k}} ; \boldsymbol{e}_{t_{k}} ; \boldsymbol{e}_{s_{k}} ; \boldsymbol{c}_{k}\right]$, where $e_{k} \in \mathbb{R}^{D_{e}}$, and $D_{e}=2 D_{l}+D_{t}+D_{s}$. Then, the $k$-th recurrent unit takes $\boldsymbol{e}_{k}$ and $\boldsymbol{h}_{k-1}$ to update the hidden state as follows:

$$
\boldsymbol{h}_{k}=f\left(\boldsymbol{W}_{r_{1}} \cdot \boldsymbol{h}_{k-1}+\boldsymbol{W}_{r_{2}} \cdot \boldsymbol{e}_{k}+\boldsymbol{b}_{r}\right)
$$

where $\boldsymbol{h}_{k} \in \mathbb{R}^{D_{h}}$ is a $D_{h}$-dimensional vector which represents the hidden state at $t_{k} ; \boldsymbol{W}_{r_{1}}$ and $\boldsymbol{W}_{r_{2}}$ are the weight matrices, and $\boldsymbol{b}_{r}$ is the bias term; $f(\cdot)$ denotes the forward function of $\mathrm{LSTM}^{3}$.

\section{Output Layer}

By performing Eq. 4 through time, we can obtain the hidden state, $h_{K-1}$, which coherently inherits the information of the trajectory until time step $t_{K-1}$. We combine it with user general preference to predict where user $u_{i}$ would visit at $t_{K}$. For this purpose, we first decode $\boldsymbol{h}_{K-1}$ into a $D_{u^{-}}$ dimensional vector, i.e., $\boldsymbol{o}_{K-1} \in \mathbb{R}^{D_{u}}$. Then, we concatenate the user embedding, $\boldsymbol{e}_{u_{i}}$, with $\boldsymbol{o}_{K-1}$ to calculate the distribution over $|L|$ locations as follows:

$$
\begin{aligned}
& \boldsymbol{o}_{K-1}=\boldsymbol{W}_{o_{1}} \cdot \boldsymbol{h}_{K-1}+\boldsymbol{b}_{o_{1}} \\
& \boldsymbol{o}_{K-1}^{\prime}=\boldsymbol{W}_{o_{2}}\left[\begin{array}{c}
\boldsymbol{o}_{K-1} \\
\boldsymbol{e}_{u}
\end{array}\right]+\boldsymbol{b}_{o_{2}} \\
& \hat{\boldsymbol{y}}_{K}^{u_{i}}=\operatorname{softmax}\left(\boldsymbol{o}_{K-1}^{\prime}\right)
\end{aligned}
$$

${ }^{3}$ The activation functions of input, forget and output gates are all sigmoid function while it is the tanh function for cell gate.
Table 1: Statistics of datasets $(\Delta t=12 h)$

\begin{tabular}{cccccc}
\hline City & Users & Locations & Check-ins & Trajectories & Words \\
\hline NY & 490 & 19253 & 77853 & 9082 & 242 \\
TK & 1499 & 33530 & 247794 & 31180 & 235 \\
SF & 170 & 7340 & 32058 & 2953 & 306 \\
\hline
\end{tabular}

where $\boldsymbol{W}_{o_{1}} \in \mathbb{R}^{D_{u} \times D_{h}}, \boldsymbol{W}_{o_{2}} \in \mathbb{R}^{|L| \times 2 D_{u}}$ are transformation matrices and $\boldsymbol{b}_{o_{1}}, \boldsymbol{b}_{o_{2}}$ are the bias terms; $\hat{\boldsymbol{y}}_{K}^{u_{i}}$ represents the probability distribution over $L$ via softmax function at time step $t_{K}$. Note that user embedding, $\boldsymbol{e}_{u_{i}}$, is only utilized in the output layer as it represents the general interest of $u_{i}$, which does not change with time.

\section{Model Optimization}

Given the training samples, the parameters are optimized by minimizing the following loss function:

$$
J=\sum_{i=1}^{|U|} \sum_{k=1}^{|L|} \boldsymbol{y}_{K}^{u_{i}}\left(l_{k}\right) \cdot \log \hat{\boldsymbol{y}}_{K}^{u_{i}}\left(l_{k}\right)+\lambda\|\Theta\|_{2}
$$

where $J$ is the Cross Entropy Loss between the predictions and ground truth; $\boldsymbol{y}_{K}^{u_{i}}$ is a one-hot vector to describe the ground truth location at $t_{K}$, i.e., $\boldsymbol{y}_{K}^{u_{i}}\left(l_{k}\right)=1$ if $u_{i}$ visited $l_{k}$ at $t_{K} ;\|\Theta\|_{2}$ is the regularization term to avoid over-fitting; $\lambda$ controls the strength of the regularization.

\section{Experiments}

We carry out experiments to investigate the following questions: (1) How will different meta-paths affect our model performance? (2) How will the time threshold and the embedding dimensionality affect our model accuracy? (3) How will our approach compare with state-of-the-art methods? (4) What is the convergence property of our model?

\section{Experimental Setup}

Data Prepossessing. Following previous work (Yao et al. 2017), users with fewer than 5 trajectories and trajectories with fewer than 3 check-ins are removed. Previous works (Yao et al. 2017; Zhang et al. 2017; Feng et al. 2018) also usually filter out inactive locations to ease the sparsity, but this filtering operation may induce the model to learn wrong transition information. Thus, no filtering is conducted to locations in our experiments. We adopt the time threshold $\Delta t=12 h$ to create high-quality trajectories, because our experiments show that it is the optimal setting (see Table 2). The statistics of datasets are summarized in Table 1.

Comparison Methods. We compare ARNN with state-ofthe-art methods: 1) UCF (Sarwar et al. 2001): is a userbased collaborative filtering by using the user-location matrix; 2) FPMC (Rendle, Freudenthaler, and Schmidt-Thieme 2010): extends Markov Chain for sequential prediction; 3) FPMC-LR (Cheng et al. 2013): extends FPMC by considering geographical constraints; 4) LSTM (Hochreiter and Schmidhuber 1997): is a popular variant model of RNN for sequential prediction; 5) ST-RNN (Liu et al. 2016): is a recent RNN-based model that incorporates temporal and geographical information; 6) SERM (Yao et al. 2017): is a stateof-the-art method combining multiple contexts in a recurrent 

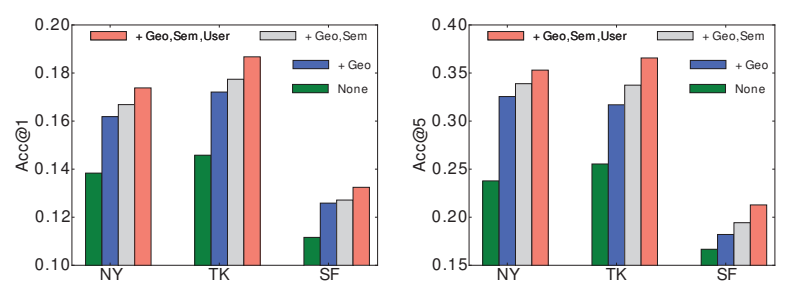

Figure 5: Performance of variants of ARNN on the three datasets when various meta-path are cumulatively incorporated.

model; 7) DeepMove (Feng et al. 2018): is a state-of-the-art recurrent model capturing multi-level influence of previous check-ins; 8) Caser (Tang and Wang 2018): is a state-ofthe-art method that models the joint effects of previous visits based on CNN.

Evaluation Metrics. Following the existing works (Zhang et al. 2017; Yao et al. 2017; Feng et al. 2018), we employ prediction accuracy (Acc@N,N=1,5,10,20) to check whether the ground-truth location appears in the top- $N$ recommendation list.

Parameter Settings. We use the earliest $70 \%$ check-ins of each user as training set (70\%), and the latest $20 \%$ as test set and remaining $10 \%$ as validation set. The parameters are turned to achieve the best results, or set as suggested by the original papers for all comparison methods. For the neighbor discovery procedure in ARNN, the distance threshold $\Delta d=2 \mathrm{~km}$, walk number walk_num $=50$ and walk length walk_len $=10$ for the meta-path based random walk. We apply a grid search to find the optimal settings for the size of user embedding $\left(D_{u}\right)$, location embedding $\left(D_{l}\right)$, timestamp embedding $\left(D_{t}\right)$ and semantics embedding $\left(D_{s}\right)$ (see Fig. 6). The 4 embedding sizes are set as the same $\left(D_{u}=D_{l}=D_{t}=D_{s}\right)$ and are $120 / 160 / 120$ for NY, TK and SF, respectively. The number of hidden units, $D_{h}$, is set as 64 for all cities. The number of recurrent layers is 1 . The epoch number is set as 20 . The learning rate is 0.01 . The regularization parameter $\lambda$ is chosen as 0.01 . The hidden state and cell state are initialized as zero. We use Stochastic Gradient Descent (Bottou 1991) and Back Propagation Through Time (Rumelhart, Hinton, and Williams 1986) to learn the parameters with a batch size of 64 .

\section{Analysis of Meta-paths}

To investigate the effect of various meta-paths, we cumulatively incorporate the selected meta-paths into neighbor discovery (see Fig. 5). The results w.r.t. Acc@1 and Acc@5, where Geo, Sem and User represent $L L, L V L$ and $L U L$, respectively. None means no meta-path is used, i.e., ARNN is degraded to LSTM. Note that similar trends can also be observed for Acc@10 and Acc@20. It can be observed that the performance becomes better as more meta-paths are incorporated. Moreover, all the ARNN variants outperform LSTM, which confirms the effectiveness of utilizing transition regularities of neighbors. In addition, meta-path $L U L$ delivers more significant enhancement than others, which
Table 2: Performance variation of ARNN with different time threshold $(\Delta t)$ on three datasets evaluated by Acc@N. The best performance is highlighted in bold.

\begin{tabular}{|c|c|c|c|c|c|c|}
\hline City & Metric & $3 h$ & $6 h$ & $12 h$ & $1 d$ & $2 d$ \\
\hline \multirow{5}{*}{ NY } & Acc@1 & 0.1816 & 0.2058 & 0.1738 & 0.1526 & 0.1220 \\
\hline & Acc@5 & 0.3437 & 0.348 & 0.3530 & 0.2943 & 0.2498 \\
\hline & Acc@10 & 0.4076 & 0.4087 & 0.4162 & 0.3317 & 0.2977 \\
\hline & Acc@20 & 0.4474 & 0.4571 & 0.4393 & 0.3544 & 0.3097 \\
\hline & $\operatorname{Pct}(\leqslant \Delta t)$ & $46.75 \%$ & $54.45 \%$ & $63.83 \%$ & $80.04 \%$ & $90.45 \%$ \\
\hline \multirow{5}{*}{ TK } & Acc@1 & 0.1811 & 0.1801 & 0.1867 & 0.1651 & 0.1321 \\
\hline & Acc@5 & 0.3448 & 0.3605 & 0.3657 & 0.3044 & 0.2283 \\
\hline & Acc@10 & 0.4121 & 0.4180 & 0.4285 & 0.3569 & 0.2637 \\
\hline & Acc@20 & 0.4625 & 0.4889 & 0.4864 & 0.4125 & 0.3392 \\
\hline & $\operatorname{Pct}(\leqslant \Delta t)$ & $58.96 \%$ & $63.89 \%$ & $71.74 \%$ & $84.33 \%$ & $92.18 \%$ \\
\hline \multirow{5}{*}{$\mathrm{SF}$} & Acc@1 & 0.0699 & 0.1297 & 0.1324 & 0.0816 & 0.0829 \\
\hline & Acc@5 & 0.1441 & 0.2010 & 0.2128 & 0.1780 & 0.1685 \\
\hline & Acc@10 & 0.1594 & 0.2318 & 0.2336 & 0.2126 & 0.2072 \\
\hline & Acc@20 & 0.1703 & 0.2431 & 0.2530 & 0.2336 & 0.2251 \\
\hline & $\operatorname{Pct}(\leqslant \Delta t)$ & $50.26 \%$ & $56.68 \%$ & $63.48 \%$ & $80.34 \%$ & $90.52 \%$ \\
\hline
\end{tabular}

verifies that the similar properties of two locations can be well captured by user preference.

With the incorporation of all meta-paths, the performance is enhanced significantly by $14.69 \%$ and $18.05 \%$ averagely w.r.t. Acc@1 and Acc@5 across the three datasets. Nevertheless, the improvements are not always significant with $L V L$ and $L L$. With $L V L$, the random walker could reach neighbors that are far away from the location. The distant locations may have no common transition patterns, as the distance of two successive check-ins is often less than $10 \mathrm{~km}$ (Feng et al. 2015). By following $L L$, although the random walker can find nearby neighbors, these neighbors (e.g., a museum) may not share similar characteristics with the location (e.g., a gym club). Despite some unsatisfactory neighbors could be retrieved, their saliences can be distinguished automatically by the attention layer.

\section{Analysis of Time Threshold}

As the time interval of two successive check-ins has critical influence on users' next location (Yao et al. 2017; Feng et al. 2015), it is necessary to investigate the impact of various settings of the time threshold $(\Delta t)$ on the accuracy of ARNN (Table 2). To better understand the results, we calculate the percentage of the successive check-in pairs that happen within various $\Delta t$, i.e., $\operatorname{Pct}(\leqslant \Delta t)=\frac{\sum_{i=1}^{|U|}\left|\left\{\left(r_{k-1}, r_{k}\right) \mid r_{k} \in H i s\left(u_{i}\right), t_{k}-t_{k-1} \leqslant \Delta t\right\}\right|}{\sum_{i=1}^{|U|}\left|\left\{\left(r_{k-1}, r_{k}\right) \mid r_{k} \in H i s\left(u_{i}\right)\right\}\right|}$, where $\operatorname{His}\left(u_{i}\right)$ represents the historical sequences of $u_{i}$, $r_{k-1}$ indicates a check-in and its following check-in is $r_{k}$.

For about $50 \%$ and $60 \%$ of successive check-ins, their time interval is smaller than $3 h$ and $6 h$, respectively, reinforcing the previous finding that the majority of successive check-ins happen within a few hours (Cheng et al. 2013). The best results are achieved with $\Delta t=12 h$, implying successive check-ins with the time interval in $6 h-12 h$ still carry effective transition information. However, the performance deteriorates when $\Delta t$ increases to $24 h$, because the time interval exceeds to a point such that wrong transition information is introduced. Overall, $\Delta t=12 h$ is a robust setting to 
Table 3: Performance of all the comparison methods on the three real-world datasets measured Acc@N. The best performance is highlighted in bold while the second best performance is marked by ' $\dagger$ '.

\begin{tabular}{r|l|llllllll|r|}
\hline City & Metric & UCF & FPMC & FPMC-LR & LSTM & ST-RNN & SERM & Caser & ARNN & Improve \\
\hline \multirow{5}{*}{ NY } & Acc@1 & 0.0010 & 0.0214 & 0.0248 & 0.1384 & 0.1292 & 0.1571 & $0.1608^{\dagger}$ & $\mathbf{0 . 1 7 3 8}$ & $8.07 \%$ \\
& Acc@5 & 0.0055 & 0.0386 & 0.0403 & 0.2378 & 0.2474 & 0.2880 & $0.3210^{\dagger}$ & $\mathbf{0 . 3 5 3 0}$ & $9.97 \%$ \\
& Acc@10 & 0.0096 & 0.0735 & 0.0783 & 0.2752 & 0.2803 & 0.3230 & $0.3767^{\dagger}$ & $\mathbf{0 . 4 1 6 2}$ & $10.50 \%$ \\
& Acc@20 & 0.0131 & 0.0872 & 0.0929 & 0.3058 & 0.3299 & 0.3437 & $0.4016^{\dagger}$ & $\mathbf{0 . 4 3 9 3}$ & $9.37 \%$ \\
\hline \hline \multirow{5}{*}{ TK } & Acc@1 & 0.0092 & 0.0196 & 0.0227 & 0.1458 & 0.1572 & 0.1608 & $0.1709^{\dagger}$ & $\mathbf{0 . 1 8 6 7}$ & $10.34 \%$ \\
& Acc@5 & 0.0197 & 0.0312 & 0.0358 & 0.2554 & 0.2443 & 0.2899 & $0.3302^{\dagger}$ & $\mathbf{0 . 3 6 5 7}$ & $8.74 \%$ \\
& Acc@10 & 0.0385 & 0.0597 & 0.0626 & 0.2989 & 0.2794 & 0.3407 & $0.38522^{\dagger}$ & $\mathbf{0 . 4 2 8 5}$ & $10.51 \%$ \\
& Acc@20 & 0.0199 & 0.0686 & 0.0753 & 0.3399 & 0.3285 & 0.3842 & $0.4387^{\dagger}$ & $\mathbf{0 . 4 8 6 4}$ & $13.09 \%$ \\
\hline \hline \multirow{5}{*}{ SF } & Acc@1 & 0.0005 & 0.0122 & 0.0167 & 0.1116 & 0.1035 & 0.1205 & $0.1220^{\dagger}$ & $\mathbf{0 . 1 3 2 4}$ & $8.54 \%$ \\
& Acc@5 & 0.0025 & 0.0245 & 0.0314 & 0.1667 & 0.1630 & $0.1949^{\dagger}$ & 0.1894 & $\mathbf{0 . 2 1 2 8}$ & $9.43 \%$ \\
& Acc@10 & 0.0045 & 0.0426 & 0.0512 & 0.1845 & 0.1860 & 0.2098 & $0.2177^{\dagger}$ & $\mathbf{0 . 2 3 3 6}$ & $7.33 \%$ \\
& Acc@20 & 0.0076 & 0.0541 & 0.0625 & 0.2009 & 0.2098 & 0.2321 & $0.2345^{\dagger}$ & $\mathbf{0 . 2 5 3 0}$ & $7.90 \%$ \\
\hline
\end{tabular}

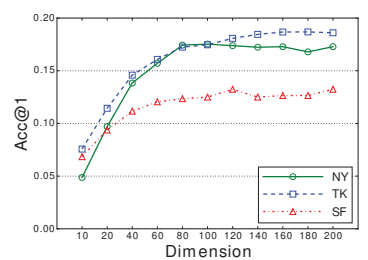

(a) Dimentionality

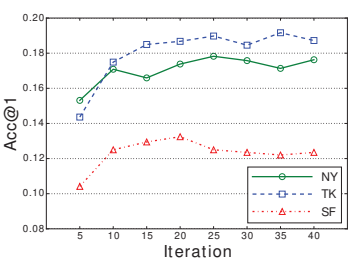

(b) Convergence

Figure 6: Analysis of Dimensionality and Convergence.

generate high-quality trajectories for model training.

\section{Analysis of Dimensionality and Convergence}

Fig. 6(a) describes the recommendation performance w.r.t. Acc@1 for various dimensionality (i.e., embedding size) with other optimal hyperparameters fixed on all the three datasets. Note similar trends can be observed for other metrics. The performance becomes stable when the dimension increases to a certain level. Besides, the optimal dimension size of Tokyo is higher than others, since there are much more locations in Tokyo. Moreover, ARNN consistently outperforms other state-of-the-art methods when its results become stable, indicating that it can well deal with the variation of dimensionality. Furthermore, to understand the convergence of ARNN, we report the performance change with respect to the number of iterations. As shown in Fig. 6(b), we can observe that ARNN can converge within 20 iterations. In summary, with strong ability to dealing with the dimensionality and fast convergence speed, ARNN is a robust approach that can be applied in practical scenarios.

\section{Comparative Results}

With optimal settings of involved parameters, we conduct experiments to evaluate our ARNN with state-of-the-art

methods. Table 3 presents the performance of all comparison methods evaluated by Acc@N. UCF performs worst since it only models user general interest without considering sequential regularity. By modeling sequential regularity, FPMC performs better than UCF. FPMC-LR outperforms FPMC by further fusing geographical information. However, the results of both Markov-based methods are still poor, as they fail to model high-order sequential regularity.

In general, RNN-based methods outperform Markovbased methods because of their capability of memorizing long-term dependencies (Hochreiter and Schmidhuber 1997). ST-RNN delivers decent results by exploiting spatial and temporal contexts. However, ST-RNN is outperformed by LSTM in some cases due to the stronger ability of LSTM to model long sequence than basic RNN. By considering semantic context, SERM performs better than ST-RNN. DeepMove adopts a similar attention-based GRU architecture applied in language translation (Luong, Pham, and Manning 2015), to capture the multi-level influence of distant checkins in the sequence. The way of integrating attention layer in ARNN is divergent from DeepMove. To explain, the attention layer in ARNN is designed to capture the transition regularities of each neighbor at each time step while DeepMove captures the impacts of previous check-ins along the entire sequence in DeepMove. In general, Caser outperforms other RNN-based methods by modeling the effects of past check-ins jointly while others model previous checkins independently. However, either the methods of learning embeddings of different contexts (ST-RNN and SERM) or modeling the complex sequential regularity (DeepMove and Caser) are deficient in solving the sparsity issue. By jointly modeling the transition regularities of various neighbors and sequential regularity, our ARNN performs the best - the average improvements are $9.48 \%, 10.26 \%, 7.89 \%$ on the three datasets, respectively. The significant improvement margins demonstrate the strong ability of ARNN in resolving the sparsity for more accurate recommendation. 


\section{Conclusion and Future Work}

In this paper, we explore the transition regularities of neighbors to resolve the sparsity issue for next location recommendation. We first design a meta-path based random walk on a novel knowledge graph to discover relevant neighbors based on heterogeneous factors. Next, we jointly model both sequential regularity and transition regularities of neighbors by proposing a novel attentional recurrent neural network framework, ARNN. Experimental results on multiple real-world datasets demonstrate the superiority of ARNN to state-of-the-art approaches. In the future, we plan to explore more useful data sources such as weather and traffic information, to provide more satisfying recommendations.

\section{Acknowledgments}

This work is partially supported by the MOE AcRF Tier 1 funding (M4011894.020) awarded to Dr. Jie Zhang.

\section{References}

Bahdanau, D.; Cho, K.; and Bengio, Y. 2014. Neural machine translation by jointly learning to align and translate. arXiv preprint arXiv:1409.0473.

Bottou, L. 1991. Stochastic gradient learning in neural networks. Proceedings of Neuro-Nimes 91(8):12.

Cheng, C.; Yang, H.; Lyu, M. R.; and King, I. 2013. Where you like to go next: Successive point-of-interest recommendation. In IJCAI, volume 13, 2605-2611.

Feng, S.; Li, X.; Zeng, Y.; Cong, G.; Chee, Y. M.; and Yuan, Q. 2015. Personalized ranking metric embedding for next new poi recommendation. In IJCAI, 2069-2075.

Feng, J.; Li, Y.; Zhang, C.; Sun, F.; Meng, F.; Guo, A.; and Jin, D. 2018. Deepmove: Predicting human mobility with attentional recurrent networks. In $W W W, 1459-1468$.

Guo, Q.; Sun, Z.; Zhang, J.; Chen, Q.; and Theng, Y.-L. 2017. Aspect-aware point-of-interest recommendation with geo-social influence. In UMAP, 17-22. ACM.

He, J.; Li, X.; Liao, L.; Song, D.; and Cheung, W. K. 2016. Inferring a personalized next point-of-interest recommendation model with latent behavior patterns. In $A A A I, 137-143$.

Hochreiter, S., and Schmidhuber, J. 1997. Long short-term memory. Neural computation 9(8):1735-1780.

Huang, L.; Ma, Y.; Wang, S.; and Liu, Y. 2019. An attention-based spatiotemporal lstm network for next poi recommendation. IEEE Transactions on Services Computing.

Koren, Y.; Bell, R.; and Volinsky, C. 2009. Matrix factorization techniques for recommender systems. Computer (8):30-37.

Li, Z.; Ding, B.; Han, J.; Kays, R.; and Nye, P. 2010. Mining periodic behaviors for moving objects. In SIGKDD, 1099-1108. ACM.

Liu, Q.; Wu, S.; Wang, L.; and Tan, T. 2016. Predicting the next location: A recurrent model with spatial and temporal contexts. In AAAI, 194-200.

Luong, M.-T.; Pham, H.; and Manning, C. D. 2015. Effective approaches to attention-based neural machine translation. arXiv preprint arXiv: 1508.04025.

Massimo, D., and Ricci, F. 2018. Harnessing a generalised user behaviour model for next-poi recommendation. In Proceedings of the 12th ACM Conference on Recommender Systems, 402-406. ACM.
Mikolov, T.; Karafiát, M.; Burget, L.; Cernockỳ, J.; and Khudanpur, S. 2010. Recurrent neural network based language model. In Interspeech, volume 2, 3.

Mnih, A., and Salakhutdinov, R. R. 2008. Probabilistic matrix factorization. In NIPS, 1257-1264.

Mnih, V.; Heess, N.; Graves, A.; et al. 2014. Recurrent models of visual attention. In NIPS, 2204-2212.

Monreale, A.; Pinelli, F.; Trasarti, R.; and Giannotti, F. 2009. Wherenext: a location predictor on trajectory pattern mining. In SIGKDD, 637-646. ACM.

Rendle, S.; Freudenthaler, C.; and Schmidt-Thieme, L. 2010. Factorizing personalized markov chains for next-basket recommendation. In $W W W, 811-820$. ACM.

Rumelhart, D. E.; Hinton, G. E.; and Williams, R. J. 1986. Learning representations by back-propagating errors. nature 323(6088):533.

Sarwar, B.; Karypis, G.; Konstan, J.; and Riedl, J. 2001. Item-based collaborative filtering recommendation algorithms. In $W W W, 285-$ 295. ACM.

Sun, Y.; Han, J.; Yan, X.; Yu, P. S.; and Wu, T. 2011. Pathsim: Meta path-based top-k similarity search in heterogeneous information networks. PVLDB 4(11):992-1003.

Sun, Z.; Guo, Q.; Yang, J.; Fang, H.; Guo, G.; Zhang, J.; and Burke, R. 2019. Research commentary on recommendations with side information: A survey and research directions. Electronic Commerce Research and Applications 37:100879.

Sutskever, I.; Vinyals, O.; and Le, Q. V. 2014. Sequence to sequence learning with neural networks. In NIPS, 3104-3112.

Tang, J., and Wang, K. 2018. Personalized top-n sequential recommendation via convolutional sequence embedding. In WSDM, 565-573. ACM.

Xie, M.; Yin, H.; Xu, F.; Wang, H.; and Zhou, X. 2016. Graphbased metric embedding for next poi recommendation. In WISE, 207-222. Springer.

Yang, D.; Zhang, D.; Zheng, V. W.; and Yu, Z. 2015. Modeling user activity preference by leveraging user spatial temporal characteristics in lbsns. IEEE Transactions on Systems, Man, and Cybernetics: Systems 45(1):129-142.

Yao, D.; Zhang, C.; Huang, J.; and Bi, J. 2017. Serm: A recurrent model for next location prediction in semantic trajectories. In CIKM, 2411-2414. ACM.

Yin, H.; Wang, W.; Wang, H.; Chen, L.; and Zhou, X. 2017. Spatial-aware hierarchical collaborative deep learning for poi recommendation. TKDE 29(11):2537-2551.

Zhang, Y.; Dai, H.; Xu, C.; Feng, J.; Wang, T.; Bian, J.; Wang, B.; and Liu, T.-Y. 2014. Sequential click prediction for sponsored search with recurrent neural networks. In AAAI, 1369-1375.

Zhang, Z.; Li, C.; Wu, Z.; Sun, A.; Ye, D.; and Luo, X. 2017. Next: a neural network framework for next poi recommendation. arXiv preprint arXiv: 1704.04576 . 